\title{
Stereospecific Deoxygenation of Phosphine Oxides with Retention of Configuration Using Triphenylphosphine as an Oxygen Acceptor
}

\author{
Hai-Chen Wu; Jin-Quan Yu*; Jonathan B. Spencer* \\ [*]Department of Chemistry, Cambridge University, Cambridge CB2 1EW (UK) \\ Fax: (+44)1223 336362 \\ Email:yu200@brandeis.edu,jbs20@cam.ac.uk
}

General methods. All reactions were carried out under a nitrogen atmosphere by using Schlenk techniques. Dry solvents were obtained from Fluka (stored over molecular sieves) or were dried by standard methods prior to use and stored under nitrogen over 4A molecular sieves. Degassed solvents were obtained by the freeze-thaw-freeze circle. Solvents were removed by evaporation on a Buchi rotary evaporator, with vacuum provided by a controlled Teflon-membrane pump. All products were dried at oil-pump vacuum (approximately $0.5 \mathrm{~mm} \mathrm{Hg}$ or better) for at least one hour before spectroscopic characterization. Thin layer chromatography (TLC) analysis was performed using Merck 60 PF254 (Merck) $0.2 \mathrm{~mm}$ plates (glass backed) and were visualized by UV. Flash chromatography was performed using Merck 9385 Kieselgel 60 silica gel.

NMR spectra were obtained on $\mathrm{CDCl}_{3}$ solution at $25^{\circ} \mathrm{C}$ using Bruker Avance DPX $400 \mathrm{MHz}$ (400 MHz for ${ }^{1} \mathrm{H}$ NMR) and Bruker Avance $500 \mathrm{MHz}$ Broadband (500 $\mathrm{MHz}$ for ${ }^{1} \mathrm{H}, 125 \mathrm{MHz}$ for ${ }^{13} \mathrm{C}$ and $202 \mathrm{MHz}$ for ${ }^{31} \mathrm{P}$ ) unless stated otherwise. Chemical shifts were reported in $\delta$ ppm referenced to the residual solvent peak $(\delta 7.24$ for ${ }^{1} \mathrm{H}$ and $\delta 77.0$ for ${ }^{13} \mathrm{C}$ ) and to an external $85 \% \mathrm{H}_{3} \mathrm{PO}_{4}$ standard for ${ }^{31} \mathrm{P}$ NMR. IR spectra were recorded using Perkin-Elmer Spectrum One FT-IR with ATR universal sampling accessory as neat solids. Wavelengths were quoted in $\mathrm{cm}^{-1}$. Mass spectra were recorded on Perkin Elmer TurboMass GC-MS (LRMS) and Micromass Q-TOF (HRMS). The parent ion and significant fragments were quoted. 
2,2'-Bis(diphenylphosphinyl)-1,1'-binaphthyl (1c) and $(R)-(+)-2,2$ '-bis(di-p-tolyl phosphinyl)-1,1'-binaphthyl (1d) were prepared according to the literature method. ${ }^{1}$ 1,2-Bis(dipentafluorophenylphosphinyl)ethane (1g) was prepared by oxidizing 1,2-bis(dipentafluorophenylphosphino)ethane which is commercially available from Strem Chemical Inc. by the previously reported procedure. $^{2}$ Tris[3,5-bis(trifluoromethyl)phenyl] phosphine oxide (1h) was prepared from tris[3,5-bis(trifluoromethyl)phenyl]phosphine using the same method. (S)-(-)-(2-Methoxyphenyl)methylphenyl phosphine [(S)-PAMP] was purchased from Digital Specialty Chemicals, Inc..

Preparation of phosphine oxides. The preparation of certain BINAP analogue diphophines has been described elsewhere. ${ }^{1}$ Herein we reported a modified procedure which is practical for small scale synthesis. $\mathrm{The} \mathrm{Ar}_{2} \mathrm{PCl}$ reagents were prepared by treating $\left(\mathrm{Et}_{2} \mathrm{~N}\right) \mathrm{PCl}_{2}$ with 2 equiv of $\mathrm{RMgBr}$ followed by treatment of the resulting $\left(\mathrm{Et}_{2} \mathrm{~N}\right) \mathrm{PAr}_{2}$ product with $\mathrm{HCl}^{3}$

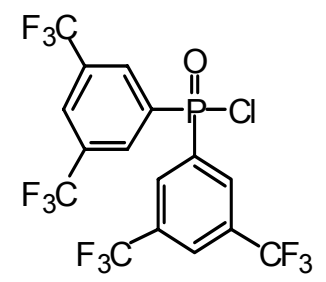

\section{Bis[3,5-bis(trifluoromethyl)phenyl]phosphinyl Chloride.}

$5.0 \mathrm{~g} \quad(0.010 \mathrm{~mol}) \quad$ Bis[3,5-bis(trifluoromethyl)phenyl]chlorophosphine was dissolved in $25 \mathrm{~mL}$ dry benzene under nitrogen atmosphere. Dry oxygen gas was conducted to bubble through the solution for 5 hours. After the bubbling was over benzene was removed under reduced pressure. The crude product (5.08 g) was collected as a white solid of about $98 \mathrm{~mol} \%$ purity by ${ }^{31} \mathrm{P}$ and ${ }^{1} \mathrm{H}$ NMR spectroscopy and used for next step without purification. ${ }^{1} \mathrm{H}$ NMR: $\delta 8.18(\mathrm{~s}, 2 \mathrm{H})$, $8.34(\mathrm{~s}, 2 \mathrm{H}), 8.37$ (s, 2H). ${ }^{13} \mathrm{C}$ NMR: $\delta 122.3\left(\mathrm{q}, J=272 \mathrm{~Hz}, \mathrm{CF}_{3}\right), 127.6(\mathrm{~d}, J=3.25$ $\mathrm{Hz}), 131.1$ (d, $J=12.6 \mathrm{~Hz}), 133.2\left(\mathrm{dq}, J=34.4 \mathrm{~Hz}, J=14.9 \mathrm{~Hz}, \mathrm{C}-\mathrm{CF}_{3}\right), 135.0$ (d, $J=$ 125.6 Hz, C-P), ${ }^{31}$ P NMR: $\delta$ 35.56. FT-IR: v 1360, 1276, 1123, 910, 844, 701, 682 $\mathrm{cm}^{-1}$. LRMS (70eV), $473[\mathrm{M}-\mathrm{Cl}]$. 

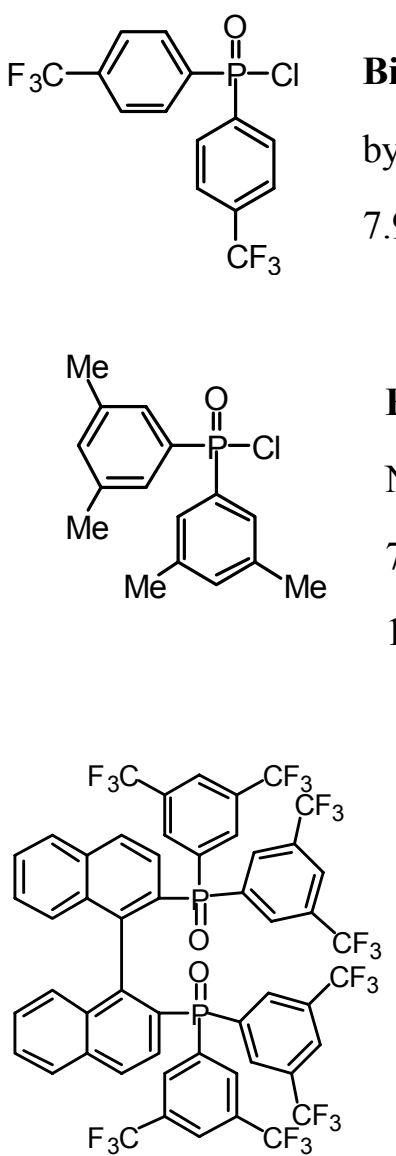

Bis[4-(trifluoromethyl)phenyl]phosphinyl Chloride. Yield by NMR: $98 \%$ (4.55 g). ${ }^{1} \mathrm{H}$ NMR: $\delta 7.68(\mathrm{~d}, J=7.6 \mathrm{~Hz}, 4 \mathrm{H})$, $7.93(\mathrm{dd}, J=13.2 \mathrm{~Hz}, J=8.0 \mathrm{~Hz}, 4 \mathrm{H}) .{ }^{31} \mathrm{P} \mathrm{NMR}: \delta 26.6$.

Bis(3,5-dimethylphenyl)phosphinyl Chloride. Yield by NMR: 98\% (3.56 g). ${ }^{1} \mathrm{H}$ NMR: $\delta 2.38$ (s, 12H), 7.22 (s, 2H), $7.48(\mathrm{~s}, 2 \mathrm{H}), 7.51(\mathrm{~s}, 2 \mathrm{H}) .{ }^{13} \mathrm{C} \mathrm{NMR}: \delta 21.2\left(\mathrm{~s}, \mathrm{CH}_{3}\right)$, 128.3-138.7. ${ }^{31} \mathrm{P}$ NMR: $\delta$ 46.9.

\begin{abstract}
Preparation of 2,2 '-Bis $\{$ bis [3,5-bis(trifluoromethyl) phenyl]\}phosphinyl-1,1'-binaphthyl (1a).

Method a: $0.092 \mathrm{~g}(3.79 \mathrm{mmol})$ Magnesium turnings were suspended in $3.0 \mathrm{~mL}$ anhydrous, degassed THF under nitrogen atmosphere. A bead of iodine was added to the above mixture and the mixture was stirred at room
\end{abstract} temperature until the color of iodine faded. To this was added dropwise a solution of 2,2'-dibromo-1,1'-binaphthyl (0.782 g, $1.90 \mathrm{mmol})$ in toluene $(12 \mathrm{~mL})$ over a period of $2 \mathrm{~h}$ at $60{ }^{\circ} \mathrm{C}$. The reaction mixture was further stirred at $75{ }^{\circ} \mathrm{C}$ for $2 \mathrm{~h}$ and was then cooled down to room temperature. To this was added dropwise a solution of bis[3,5-bis(trifluoromethyl)phenyl]phosphinyl chloride (2.32 g, $4.55 \mathrm{mmol})$ in THF (5 $\mathrm{mL}$ ) during $5 \mathrm{~min}$. After the addition was completed, the mixture was further stirred at $60{ }^{\circ} \mathrm{C}$ for $3 \mathrm{~h}$ and then cooled down to room temperature. To the solution was added 5 $\mathrm{mL}$ of $10 \%$ aqueous $\mathrm{NH}_{4} \mathrm{Cl}$ solution and the mixture was stirred for another $10 \mathrm{~min}$ at ambient temperature. The organic layer was separated, washed successively with 10 $\mathrm{mL}$ of saturated $\mathrm{NaHCO}_{3}$ solution and two $10 \mathrm{~mL}$ portions of water and then dried over anhydrous $\mathrm{MgSO}_{4}$. Solvent was removed under reduced pressure and the residue was purified by flash chromatography on silica gel (diethyl ether/hexane 1:6) to afford $0.455 \mathrm{~g}$ of the title compound as colourless needle. Yield: $20 \%{ }^{1} \mathrm{H}$ NMR: $\delta 6.83$ (d, $J=$ $8.8 \mathrm{~Hz} 2 \mathrm{H}), 7.09$ (dd, $J=7.2 \mathrm{~Hz}, J=7.2 \mathrm{~Hz}, 2 \mathrm{H}), 7.39$ (dd, $J=8.8 \mathrm{~Hz}, J=12.4 \mathrm{~Hz}, 2 \mathrm{H})$, 
$7.47(\mathrm{dd}, J=6.8 \mathrm{~Hz}, J=6.8 \mathrm{~Hz}, 2 \mathrm{H}), 7.82(\mathrm{~m}, 4 \mathrm{H}), 7.93(\mathrm{~m}, 8 \mathrm{H}), 8.21(\mathrm{~d}, J=11.2 \mathrm{~Hz}$, 2H). ${ }^{13} \mathrm{C}$ NMR: $\delta 122.6$ (q, $J=272 \mathrm{~Hz}, \mathrm{CF}_{3}$ ), 125.8-141.8. ${ }^{31}$ P NMR: $\delta 24.9$. FT-IR: $v$ $1360,1276,1181,1097,908,814,747,705,681 \mathrm{~cm}^{-1}$. HRMS $(70 \mathrm{eV})$, calcd $[\mathrm{M}+\mathrm{Na}]$ for $\mathrm{C}_{52} \mathrm{H}_{24} \mathrm{O}_{2} \mathrm{~F}_{24} \mathrm{P}_{2} \mathrm{Na}$ : 1221.0766; found: 1221.0873 , calcd [M+H] for $\mathrm{C}_{52} \mathrm{H}_{25} \mathrm{O}_{2} \mathrm{~F}_{24} \mathrm{P}_{2}$ : 1199.1035; found: 1199.1050

Method b: $0.118 \mathrm{~g}(4.86 \mathrm{mmol})$ Magnesium turnings were suspended in $5.0 \mathrm{~mL}$ anhydrous, degassed THF under nitrogen atmosphere. A bead of iodine was added to the above mixture and the mixture was stirred at room temperature until the color of iodine faded. To this was added dropwise a solution of 2,2'-dibromo-1,1'-binaphthyl $(1.00 \mathrm{~g}, 2.43 \mathrm{mmol})$ in toluene $(15 \mathrm{~mL})$ over a period of $2 \mathrm{~h}$ at $60{ }^{\circ} \mathrm{C}$. The reaction mixture was further stirred at $75{ }^{\circ} \mathrm{C}$ for $2 \mathrm{~h}$ and was then cooled down to room temperature. To this was added dropwise a solution of bis[3,5-bis(trifluoromethyl)phenyl]bromophosphine $(3.13 \mathrm{~g}, 5.83 \mathrm{mmol})$ in THF $(8 \mathrm{~mL})$ during $30 \mathrm{~min}$. After the addition was completed, the mixture was further stirred at 60 ${ }^{\circ} \mathrm{C}$ for $2 \mathrm{~h}$ and then cooled down to room temperature. To the solution was added $5 \mathrm{~mL}$ of $10 \%$ aqueous $\mathrm{NH}_{4} \mathrm{Cl}$ solution and excess $\mathrm{H}_{2} \mathrm{O}_{2}$. The mixture was stirred for another $30 \mathrm{~min}$ at ambient temperature. Diethyl ether $(30 \mathrm{~mL})$ was then added and the organic layer was separated, washed successively with $10 \mathrm{~mL}$ of saturated $\mathrm{NaHCO}_{3}$ solution and two $10 \mathrm{~mL}$ portions of water and then dried over anhydrous $\mathrm{MgSO}_{4}$. Solvent was removed under reduced pressure and the residue was purified by flash chromatography on silica gel (diethyl ether/hexane 1:6) to afford the phosphine oxide $2.04 \mathrm{~g}$ in $70 \%$ yield. All the analytical data are identical as method $\mathbf{a}$.

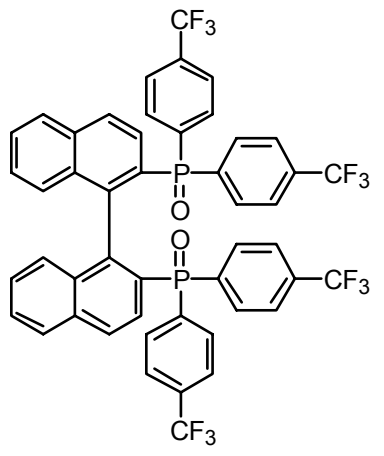

2,2'-Bis [bis(4-triflu oromethylph enyl)] phosphinyl -1,1'-binaphthyl (1b). Yield: 25\% (0.52 g). ${ }^{1} \mathrm{H}$ NMR: $\delta$ $6.69(\mathrm{~d}, J=8.5 \mathrm{~Hz} 2 \mathrm{H}), 6.83$ (t, $J=8.2 \mathrm{~Hz}, 2 \mathrm{H}), 7.36-7.44$ (m, 4H), 7.51 (d, 6.2 Hz, 8H), 7.59 (dd, $J=8.2 \mathrm{~Hz}, J=1.8$ $\mathrm{Hz}, 4 \mathrm{H}), 7.84-7.88(\mathrm{~m}, 6 \mathrm{H}), 7.93(\mathrm{dd}, J=8.5 \mathrm{~Hz}, J=2.5$ 
Hz, 2H). ${ }^{13} \mathrm{C}$ NMR: $\delta 123.5$ (dq, $J=271 \mathrm{~Hz}, J=5.5 \mathrm{~Hz}$ ), 124.9-142.6. ${ }^{31} \mathrm{P}$ NMR: $\delta$ 27.1. FT-IR: $v$ 1399, 1322, 1121, 1061, 1018, 836, 816, 744, 713, $701 \mathrm{~cm}^{-1}$. HRMS (70eV), calcd [M+Na] for $\mathrm{C}_{48} \mathrm{H}_{28} \mathrm{O}_{2} \mathrm{~F}_{12} \mathrm{P}_{2} \mathrm{Na}$ : 949.1265; found: 949.1291. Calcd $[\mathrm{M}+\mathrm{H}]$ for $\mathrm{C}_{48} \mathrm{H}_{29} \mathrm{O}_{2} \mathrm{~F}_{12} \mathrm{P}_{2}$ : 927.1446; found: 927.1526 .

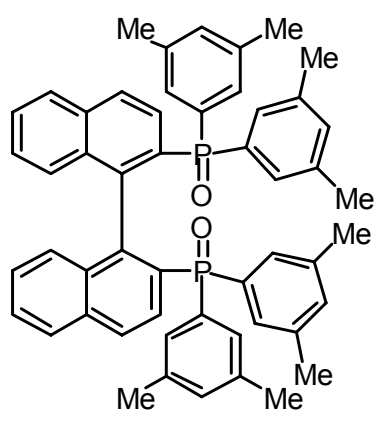

\section{2,2'-Bis[bis(3,5-dimethylphenyl)] phosphinyl -1,1'-}

binaphthyl (1e). The general procedure was followed by extending the coupling reaction time to $16 \mathrm{~h}$. Yield: $71 \%$ (1.24 g). ${ }^{1} \mathrm{H}$ NMR $\left(\mathrm{CD}_{2} \mathrm{Cl}_{2}\right): \delta 2.13(\mathrm{~s}, 12 \mathrm{H}), 2.23(\mathrm{~s}$, 12H), 6.85-6.93 (m, 4H), 6.99 (s, 2H), 7.07 (s, 2H), 7.11 (s, 2H), 7.14 (s, 2H), 7.23 (s, 2H), 7.25 (s, 2H), 7.42 (ddd, $J=8.0 \mathrm{~Hz}, J=8.0 \mathrm{~Hz}$ and $\mathrm{J}=1.1 \mathrm{~Hz}, 2 \mathrm{H}), 7.56(\mathrm{dd}, J=$ $11.6 \mathrm{~Hz}, J=8.6 \mathrm{HZ}, 2 \mathrm{H}), 7.84-7.88(\mathrm{~m}, 4 \mathrm{H}) .{ }^{13} \mathrm{C} \mathrm{NMR}\left(\mathrm{CD}_{2} \mathrm{Cl}_{2}\right): \delta 20.8\left(\mathrm{~s}, \mathrm{CH}_{3}\right)$, $20.9\left(\mathrm{~s}, \mathrm{CH}_{3}\right), 125.6$ - 142.4. ${ }^{31} \mathrm{P}$ NMR $\left(\mathrm{CD}_{2} \mathrm{Cl}_{2}\right): \delta$ 28.62. FT-IR: $v 2914,1416,1198$, $1123,851,812,744 \mathrm{~cm}^{-1}$.<smiles>O=P(c1cc(C(F)(F)F)cc(C(F)(F)F)c1)(c1cc(C(F)(F)F)cc(C(F)(F)F)c1)c1ccc2ccccc2c1-c1cccc2ccccc12</smiles>

2-Bis $\{$ bis[3,5-bis(trifluoromethyl)phenyl]\}phosphinyl1,1' - binaphthyl (1f). Yield: 23\% (0.26 g). ${ }^{1} \mathrm{H}$ NMR: $\delta$ $6.90(\mathrm{~d}, J=8.4 \mathrm{~Hz}, 1 \mathrm{H}), 7.16(\mathrm{dd}, J=7.2 \mathrm{~Hz}, J=7.2 \mathrm{~Hz}$, 1H), 7.24-7.38 (m, 4H), 7.53-7.67 (m, $6 \mathrm{H}), 7.71$ (d, $J=$ $8.2 \mathrm{~Hz}, 1 \mathrm{H}), 7.76(\mathrm{dd}, J=7.0 \mathrm{~Hz}, J=1.1 \mathrm{~Hz}, 1 \mathrm{H})$, $7.99-8.03(\mathrm{~m}, 2 \mathrm{H}), 8.09(\mathrm{dd}, J=8.6 \mathrm{~Hz}, J=2.5 \mathrm{~Hz}, 1 \mathrm{H}), 8.23(\mathrm{~s}, 1 \mathrm{H}), 8.25(\mathrm{~s}, 1 \mathrm{H}) .{ }^{13} \mathrm{C}$ NMR: $\delta 12122.4\left(\mathrm{q}, J=272 \mathrm{~Hz}, \mathrm{CF}_{3}\right), 122.7\left(\mathrm{q}, J=272 \mathrm{~Hz}, \mathrm{CF}_{3}\right), 124.4-145.8 .{ }^{31} \mathrm{P}$ NMR: $\delta 22.56$ (s). FT-IR: $v$ 1363, 1279, 1132, 909, 844, 701, $683 \mathrm{~cm}^{-1}$. HRMS (70eV), calcd $\mathrm{M}$ for $\mathrm{C}_{36} \mathrm{H}_{19} \mathrm{~F}_{12} \mathrm{PO}$ : 726.0982; found: 726.0963 .

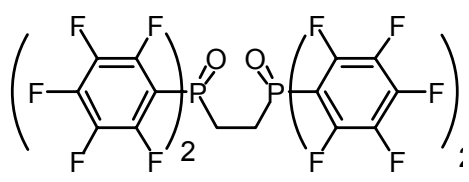

Preparation of 1,2-Bis(dipentafluorophenylphos phinyl)ethane (1g). To a solution of $0.5 \mathrm{~g}(0.66$ mmol) 1,2-bis(dipentafluorophenylphosphino)ethane in $50 \mathrm{~mL}$ of THF was added $3 \mathrm{~mL}(26.4$ 
mmol) of $30 \% \mathrm{H}_{2} \mathrm{O}_{2}$. The reaction mixture was stirred at room temperature until TLC showed the absence of starting material. Solvent was removed under reduced pressure and the residue was recrystallized from dichloromethane and cyclohexane to afford 0.48 g pure product. Yield: 92\%. ${ }^{1} \mathrm{H}$ NMR: $\delta 3.04$ (s, 4H). ${ }^{13} \mathrm{C}$ NMR: $\delta 25.6\left(\mathrm{~m},-\mathrm{CH}_{2}-\right)$, 106.7, 138.0, 144.0, 147.1. ${ }^{31}$ P NMR: $\delta 19.16$ (s). FT-IR: v 3198, 1478, 1362, 1279, 1176, 1127, 980, 904, 702, $682 \mathrm{~cm}^{-1}$. HRMS $(70 \mathrm{eV})$, calcd $[\mathrm{M}+\mathrm{Na}]$ for $\mathrm{C}_{26} \mathrm{H}_{4} \mathrm{O}_{2} \mathrm{~F}_{20} \mathrm{P}_{2} \mathrm{Na}$ : 812.9259; found: 812.9244.

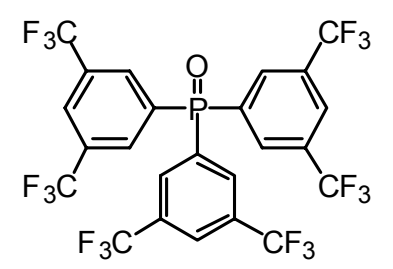

Tris[3,5-bis(trifluoromethyl)phenyl]phosphine oxide

(1h). The same procedure was followed as 1g. Yield: 96\% (0.88 g). ${ }^{1} \mathrm{H}$ NMR: $\delta 8.14(\mathrm{~s}, 3 \mathrm{H}), 8.16(\mathrm{~s}, 3 \mathrm{H}), 8.21(\mathrm{~s}$, $3 \mathrm{H}) .{ }^{13} \mathrm{C} \mathrm{NMR}: \delta 122.4\left(\mathrm{q}, J=272 \mathrm{~Hz}, \mathrm{CF}_{3}\right), 127.4(\mathrm{~d}, J=$ $3.1 \mathrm{~Hz}), 131.7(\mathrm{~d}, J=10.6 \mathrm{~Hz}), 132.9(\mathrm{~d}, J=105 \mathrm{~Hz}), 133.4(\mathrm{dq}, J=12.5 \mathrm{~Hz}, J=$ $34.3 \mathrm{~Hz},{\left.\mathrm{C}-\mathrm{CF}_{3}\right) .}^{31} \mathrm{P}$ NMR: $\delta$ 22.28. FT-IR: $v \quad 1362,1278,1173,1127,904,838$, 701, $681 \mathrm{~cm}^{-1}$. HRMS (70eV), calcd [M+Na] for $\mathrm{C}_{24} \mathrm{H}_{9} \mathrm{OF}_{18} \mathrm{PNa}$ : 709.0001; found: 709.0021 .

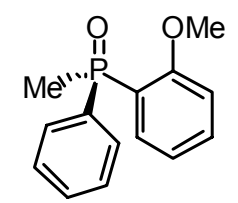

$(R)-(+)-(2-M e t h o x y p h e n y l) m e t h y l p h e n y l$ phosphine oxide [(R)-PAMPO]. The same procedure was followed as 1g. Yield: 99\% (0.36 g), 99.5\% e.e.. ${ }^{1} \mathrm{H}$ NMR: $\delta 2.05(\mathrm{~d}, J=14.1 \mathrm{~Hz}, 3 \mathrm{H})$, 3.69 (s, 3H), $6.86(\mathrm{dd}, J=8.3 \mathrm{~Hz}, J=5.6 \mathrm{~Hz}, 1 \mathrm{H}), 7.07$ (dt, $J=7.5$ $\mathrm{Hz}, J=1.6 \mathrm{~Hz}, 1 \mathrm{H}), 7.39(\mathrm{~m}, 2 \mathrm{H}), 7.46(\mathrm{~m}, 2 \mathrm{H}), 7.71$ (m, 2H), 7.94 (ddd, $J=13.3 \mathrm{~Hz}$, $J=7.6 \mathrm{~Hz}, J=1.7 \mathrm{~Hz}, 1 \mathrm{H}) .{ }^{13} \mathrm{C}$ NMR: $\delta 16.1(\mathrm{~d}, J=74.8 \mathrm{~Hz}), 55.3,110.9(\mathrm{~d}, J=6.5$ Hz), 121.0 (d, $J=11 \mathrm{~Hz}), 121.5$ (d, $J=99.1 \mathrm{~Hz}), 128.2$ (d, $J=12.1 \mathrm{~Hz}), 130.2$ (d, $J=$ $10.1 \mathrm{~Hz}), 131.2$ (d, $J=2.5 \mathrm{~Hz}), 133.9$ (d, $J=2.1 \mathrm{~Hz}), 135.0$ (d, $J=103 \mathrm{~Hz}), 159.9$ (d, $J=4.1 \mathrm{~Hz}) .{ }^{31} \mathrm{P}$ NMR: $\delta$ 28.94. $[\alpha]^{25}{ }_{\mathrm{D}}+25.8(c 1.0 \mathrm{MeOH})$. (lit. $[\alpha]^{25}{ }_{\mathrm{D}}+25.9(c 1.0$ $\mathrm{MeOH}))^{4}$

General procedure for the reduction of phosphine oxides using triphenylphosphine. In a $20 \mathrm{~mL}$ pressure tube $0.20 \mathrm{mmol}$ phosphine oxide and 0.80 mmol triphenylphosphine were dissolved in the $6 \mathrm{~mL}$ mixture of degassed THF and 
toluene (1:1) under nitrogen atmosphere. To the solution was added $0.80 \mathrm{~mL}(8.0$ mmol) trichlorosilane at room temperature. The reaction mixture was then stirred at $100{ }^{\circ} \mathrm{C}$. After TLC showed the absence of starting material and the mono-reduced product, the mixture was cooled down to ambient temperature and diluted with diethyl ether or dichloromethane (for $3 \mathrm{a}, 4 \mathrm{a}, 5 \mathrm{a}$ ). To the solution was added $10 \mathrm{~g}$ ice and $10 \mathrm{~mL} 20 \% \mathrm{NaOH}$ solution. The mixture was transferred to a separating funnel and shaked for $10 \mathrm{~min}$. (For 3a, 4a and 5a the solution was heated to $50{ }^{\circ} \mathrm{C}$ to help layers separate.) The organic layer was separated and washed successively with saturated $\mathrm{NaHCO}_{3}$, brine and water. The solution was then dried over anhydrous $\mathrm{Na}_{2} \mathrm{SO}_{4}$ and solvent removed under reduced pressure. The residue was purified by flash chromatography on silica gel (diethyl ether/hexane 1:10) to afford the title phosphine.

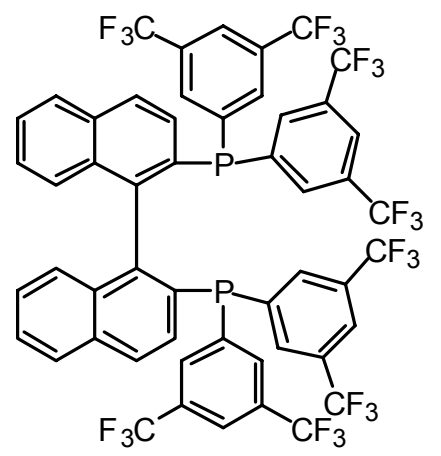

2,2'-Bis $\{$ bis[3,5-bis(trifluoromethyl)phenyl]\}phosp hino-1,1'-binaphthyl (2a). Reaction time: 96 h. Yield: 90\% (0.21 g). ${ }^{1} \mathrm{H}$ NMR: $\delta 6.88(\mathrm{~d}, J=8.5 \mathrm{~Hz}, 2 \mathrm{H})$, $7.22(\mathrm{dd}, J=7.4 \mathrm{~Hz}, J=7.4 \mathrm{~Hz}, 2 \mathrm{H}), 7.36(\mathrm{~d}, J=8.6$ $\mathrm{Hz}, 2 \mathrm{H}), 7.49-7.52(\mathrm{~m}, 8 \mathrm{H}), 7.59(\mathrm{dd}, J=7.2 \mathrm{~Hz}, J=$ $7.2 \mathrm{~Hz}, 2 \mathrm{H}), 7.70(\mathrm{~s}, 2 \mathrm{H}), 7.80(\mathrm{~s}, 2 \mathrm{H}), 8.0(\mathrm{~d}, J=8.2$ $\mathrm{Hz}, 2 \mathrm{H}), 8.08(\mathrm{~d}, J=8.5 \mathrm{~Hz}, 2 \mathrm{H}) .{ }^{13} \mathrm{C} \mathrm{NMR}: \delta 122.7$ (q, $\left.J=272 \mathrm{~Hz}, \mathrm{CF}_{3}\right), 122.8\left(\mathrm{q}, J=272 \mathrm{~Hz}, \mathrm{CF}_{3}\right), 122.3-146.4 .{ }^{31} \mathrm{P}$ NMR: $\delta-12.62$. FT-IR: $v 1352,1276,1174,1129,896,844,816,747,703,682 \mathrm{~cm}^{-1}$. HRMS (70eV), calcd $[\mathrm{M}+\mathrm{H}]$ for $\mathrm{C}_{52} \mathrm{H}_{25} \mathrm{~F}_{24} \mathrm{P}_{2}$ : 1167.1043; found: 1167.1137 .

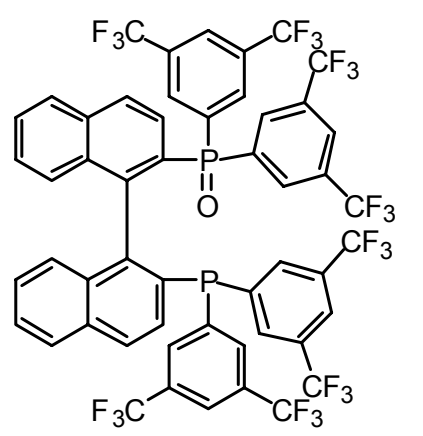

\section{2-Bis[3,5-bis(trifluoromethyl)phenyl]phosphinyl-2'-b} is $\left[3,5\right.$-bis(trifluoromethyl)phenyl] phosphino- $1,1^{\prime}$ binaphthyl (3a) was also characterised by spectroscopic measurement. ${ }^{1} \mathrm{H}$ NMR: $\delta 6.36(\mathrm{~d}, 8.4 \mathrm{~Hz}$, $1 \mathrm{H}), 6.80(\mathrm{t}, J=7.3 \mathrm{~Hz}, 1 \mathrm{H}), 6.87$ (d, $J=8.6 \mathrm{~Hz}, 1 \mathrm{H})$, $7.15(\mathrm{dd}, J=7.6 \mathrm{~Hz}, J=7.6 \mathrm{~Hz}, 1 \mathrm{H}), 7.25$ (d, $J=6.8$ 
$\mathrm{Hz}, 2 \mathrm{H}), 7.35-7.38(\mathrm{~m}, 2 \mathrm{H}), 7.46-7.49(\mathrm{~m}, 2 \mathrm{H}), 7.56-7.60(\mathrm{~m}, 3 \mathrm{H}), 7.64(\mathrm{~d}, J=8.2$ $\mathrm{Hz}, 1 \mathrm{H}), 7.71(\mathrm{~s}, 1 \mathrm{H}), 7.84(\mathrm{~d}, J=8.6 \mathrm{~Hz}, 1 \mathrm{H}), 7.90(\mathrm{~s}, 1 \mathrm{H}), 8.08(\mathrm{~m}, 3 \mathrm{H}), 8.14(\mathrm{dd}, J$ $=8.6 \mathrm{~Hz}, J=2.6 \mathrm{~Hz}, 1 \mathrm{H}), 8.37(\mathrm{~d}, J=11.0 \mathrm{~Hz}, 2 \mathrm{H}) .{ }^{13} \mathrm{C}$ NMR: $\delta . ~ \cdot{ }^{31} \mathrm{P}$ NMR: $\delta-11.68$ (s), 21.13 (d). FT-IR: $v$ 2924, 2852, 1736, 1362, 1280, 1185, 1138, $682 \mathrm{~cm}^{-1}$. HRMS (70eV), calcd [M+Na] for $\mathrm{C}_{52} \mathrm{H}_{24} \mathrm{OF}_{24} \mathrm{P}_{2} \mathrm{Na}$ : 1205.0811; found 1205.0693 .

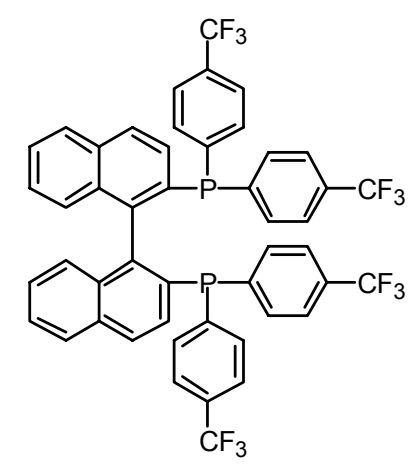

\section{2,2'-Bis[bis (4-trifluoromethylphenyl)] phosphino}

-1,1'-binaphthyl (2b). Reaction time: 18 h. Yield: 88\% (0.16 g). ${ }^{1} \mathrm{H}$ NMR: $\delta 6.81(\mathrm{~d} J=8.5 \mathrm{~Hz}, 2 \mathrm{H}), 7.02-7.06$ $(\mathrm{m}, 2 \mathrm{H}), 7.15-7.20(\mathrm{~m}, 8 \mathrm{H}), 7.39-7.42(\mathrm{~m}, 10 \mathrm{H})$, 7.45-7.48 (m, 2H), $7.91(\mathrm{~d}, J=8.2 \mathrm{~Hz}, 2 \mathrm{H}), 7.98$ (d, $J=$ $8.5 \mathrm{~Hz}, 2 \mathrm{H}) .{ }^{13} \mathrm{C}$ NMR: $\delta 123.8\left(\mathrm{q}, J=271 \mathrm{~Hz}, \mathrm{CF}_{3}\right)$, 124.9-145.7. ${ }^{31}$ P NMR: $\delta$-14.27(s). FT-IR: $v$ 1606, 1398, 1322, 1167, 1117, 1060, 1015, 833, 818, 743, 702, $695 \mathrm{~cm}^{-1}$. HRMS (70eV), calcd $[\mathrm{M}+\mathrm{H}]$ for $\mathrm{C}_{48} \mathrm{H}_{25} \mathrm{~F}_{12} \mathrm{P}_{2}$ : 895.1547; found 895.1569.

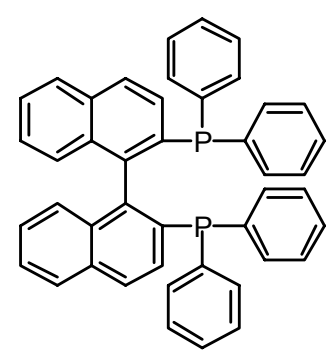

\section{2,2'-Bis(diphenylphosphino)-1,1'-binaphthyl (2c).}

Reaction time: 3 h. Yield: 95\%(0.12 g). ${ }^{1} \mathrm{H}$ NMR: $\delta 6.85(\mathrm{~d}$, $J=8.4 \mathrm{~Hz}, 2 \mathrm{H}), 6.93(\mathrm{~m}, 2 \mathrm{H}), 7.07-7.25(\mathrm{~m}, 20 \mathrm{H}), 7.37$ (m, 2H), $7.47(\mathrm{dd}, J=8.5 \mathrm{~Hz}, J=2.2 \mathrm{~Hz}, 2 \mathrm{H}), 7.85(\mathrm{~d}, J=8.2$ Hz, 2H), 7.91 (d, $J=8.5 \mathrm{~Hz}, 2 \mathrm{H}) .{ }^{31} \mathrm{P}$ NMR: $\delta-14.79$.

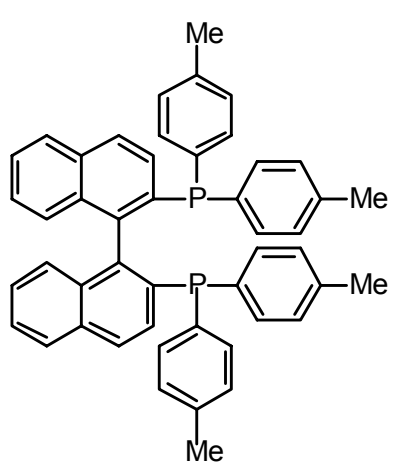

\section{(R)-(+)-2,2'-Bis(di-p-tolylphosphino)-1,1'-binaphthyl}

(2d). Reaction time: 2 h. Yield: $92 \%(0.125 \mathrm{~g}) .[\alpha]^{25}$ $+172.0^{\circ}$ (c 0.56 , benzene) (lit. $[\alpha]_{\mathrm{D}}^{24}+174.0^{\circ}$ (c 0.886 , benzene)). ${ }^{1} \mathrm{H}$ NMR: $\delta 2.28$ (s, 6H), $2.29(\mathrm{~s}, 6 \mathrm{H}), 6.88(\mathrm{~d}$, $J=8.5 \mathrm{~Hz}, 2 \mathrm{H}), 6.95-7.03(\mathrm{~m}, 18 \mathrm{H}), 7.37-7.40$ (m, 2H), $7.50(\mathrm{dd}, J=8.5 \mathrm{~Hz}, J=1.9 \mathrm{~Hz}, 2 \mathrm{H}), 7.85$ (d, $J=8.1 \mathrm{~Hz}$, 2H), $7.89(\mathrm{~d}, J=8.5 \mathrm{~Hz}, 2 \mathrm{H}) .{ }^{13} \mathrm{C}$ NMR: $\delta 21.19,21.25$,

125.73-145.32. ${ }^{31}$ P NMR: $\delta-16.24$. 


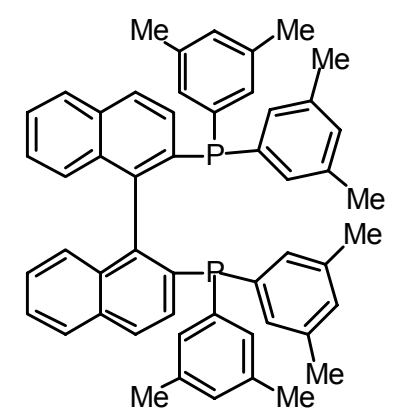

2,2'-Bis[bis(3,5-dimethylphenyl)]phosphino-1,1'-bina ph-thyl (2e). Reaction time: 2 h. Yield: 96\% (0.135 g). ${ }^{1} \mathrm{H}$ NMR: $\delta 2.14(\mathrm{~s}, 12 \mathrm{H}), 2.19(\mathrm{~s}, 12 \mathrm{H}), 6.73-6.77$ (m, 4H), $6.82(\mathrm{~s}, 2 \mathrm{H}), 6.89$ (s, 2H), 6.93 (d, $J=8.5 \mathrm{~Hz}, 2 \mathrm{H}), 7.09$ $(\mathrm{dd}, J=7.3 \mathrm{~Hz}, J=7.2 \mathrm{~Hz}, 2 \mathrm{H}), 7.46(\mathrm{dd}, J=7.2 \mathrm{~Hz}, J=$ $7.2 \mathrm{~Hz}, 2 \mathrm{H}), 7.54$ (d, $J=8.5 \mathrm{~Hz}, 2 \mathrm{H}), 7.92-7.95(\mathrm{~m}, 4 \mathrm{H})$.

${ }^{13}$ C NMR: $\delta$ 20.89, 20.96, 125.60-145.64. ${ }^{31}$ P NMR: $\delta-14.81$.

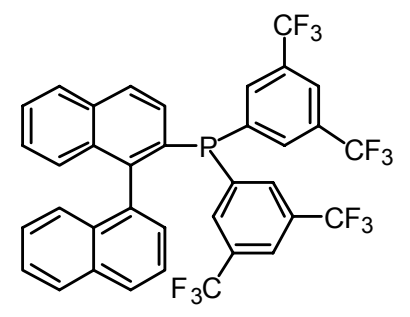

\section{2-Bis $\{$ bis[3,5-bis(trifluoromethyl)phenyl]\}phosphino-1,}

1 'binaphthyl (2f). Reaction time: 20 h. Yield: 88\% $(0.125 \mathrm{~g}) .{ }^{1} \mathrm{H}$ NMR: $\delta 6.93(\mathrm{~d}, J=8.4 \mathrm{~Hz}, 1 \mathrm{H}), 7.14-7.17$ (m, 1H), 7.26-7.46 (m, 5H), $7.50(\mathrm{~d}, J=6.5 \mathrm{~Hz}, 2 \mathrm{H})$, 7.57-7.61 (m, 2H), $7.67(\mathrm{~s}, 1 \mathrm{H}), 7.68(\mathrm{~s}, 1 \mathrm{H}), 7.74(\mathrm{~s}, 1 \mathrm{H})$, 7.90-8.02 (m, 5H). ${ }^{13} \mathrm{C}$ NMR: $\delta 122.8$ (q, $J=273 \mathrm{~Hz}, \mathrm{CF}_{3}$ ), 123.0 (q, $J=273 \mathrm{~Hz}, \mathrm{CF}_{3}$ ), 122.85-146.61. ${ }^{31}$ P NMR: $\delta$-11.06. FT-IR: $v$ 1351, 1274, 1173, 1118, 1095, 895, 844, 803, 782, 745, 703, $681 \mathrm{~cm}^{-1}$. HRMS (70eV), calcd M for $\mathrm{C}_{36} \mathrm{H}_{19} \mathrm{~F}_{12} \mathrm{P}: 710.1033$; found: 710.1001 .

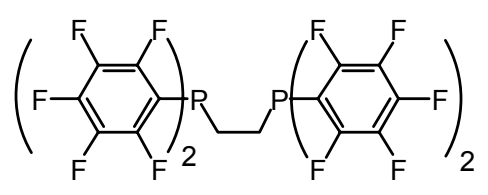

1,2-Bis(dipentafluorophenylphosphino) ethane (2g). Reaction time: 4 h. Yield: $90 \%\left(0.14\right.$ g). ${ }^{1} \mathrm{H}$ NMR: $\delta 2.58(\mathrm{t}, J=6.95 \mathrm{~Hz}, 4 \mathrm{H}) .{ }^{13} \mathrm{C}$ NMR: $\delta$ 20.20, 107.58-148.70. ${ }^{31}$ P NMR: $\delta$-43.41 (m). FT-IR: v 1640, 1516, 1466, 1384, 1291 , $1086,970,840,744,684 \mathrm{~cm}^{-1}$.

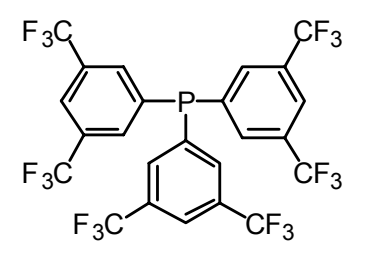

Tris[3,5-bis(trifluoromethyl)phenyl]phosphine

(2h). Reaction time: 15 h. Yield: 93\%(0.13 g). ${ }^{1} \mathrm{H}$ NMR: $\delta 7.73$ (s, 3H), 7.74 (s, 3H), 7.98 (s, 3H). ${ }^{31}$ P NMR: $\delta$-2.89. FT-IR: v $1355,1277,1172,1122,900,840,701,681 \mathrm{~cm}^{-1}$. 


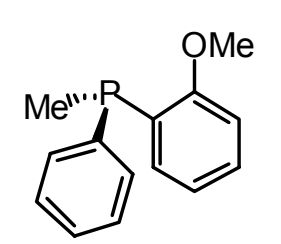

\section{(S)-(-)-(2-Methoxyphenyl)ethylphenylphosphine [(S)-PAMP].}

This reaction was conducted at the temperature of $80^{\circ} \mathrm{C}$ using $0.80 \mathrm{mmol}(S)$-PAMPO. Reaction time: $3 \mathrm{~h}$. Yield: 95\% (0.175 g).

${ }^{1} \mathrm{H}$ NMR: $\delta 1.62(\mathrm{~d}, J=4.1 \mathrm{~Hz}, 3 \mathrm{H}), 3.81(\mathrm{~s}, 3 \mathrm{H}), 6.88(\mathrm{dd}, J=$ $8.2 \mathrm{~Hz}, J=4.1 \mathrm{~Hz}, 1 \mathrm{H}), 6.94$ (t, $J=7.4 \mathrm{~Hz}, 1 \mathrm{H}), 7.07$ (m, H), 7.31-7.39 (m, 4H), 7.49 (m, 2H). ${ }^{13} \mathrm{C}$ NMR: $\delta 11.1(\mathrm{~d}, J=13.2 \mathrm{~Hz}), 55.5,110.1(\mathrm{~d}, J=33.6 \mathrm{~Hz}), 120.9,128.2$ (d, $J=6.8 \mathrm{~Hz}), 128.4,129.8,131.5$ (d, $J=3.4 \mathrm{~Hz}), 132.6$ (d, $J=19.4 \mathrm{~Hz}), 161.0$ (d, $J$ $=13.3 \mathrm{~Hz}) \cdot{ }^{31}$ P NMR: $\delta$-36.04. $[\alpha]^{25} \mathrm{D}-42.7$ (c $\left.1.3 \mathrm{MeOH}\right)$, e.e. $99 \%$ compared with literature $\left([\alpha]^{25}-38.3(c 1.7 \mathrm{MeOH}), 89 \%\right.$ e.e. $){ }^{5}$

\section{General procedure for the reduction of phosphine oxides using triethylphosphite.}

In a $20 \mathrm{~mL}$ pressure tube $0.24 \mathrm{~g}(0.20 \mathrm{mmol})$ phosphine oxide $1 \mathrm{a}$ and $0.33 \mathrm{~g}(2.00$ mmol) triethylphosphite were dissolved in the $6 \mathrm{~mL}$ mixture of degassed THF and Toluene (1:1) under nitrogen atmosphere. To the solution was added $0.80 \mathrm{~mL}(8.0$ mmol) trichlorosilane at room temperature. The reaction mixture was then stirred at $100{ }^{\circ} \mathrm{C}$. After TLC showed the absence of starting material and the mono-reduced product ( 88 hours), the mixture was cooled down to ambient temperature and diluted with diethyl ether. To the solution was added $10 \mathrm{~g}$ ice and $10 \mathrm{~mL} 20 \% \mathrm{NaOH}$ solution. The mixture was transferred to a separating funnel and shaked for $10 \mathrm{~min}$. The organic layer was separated and washed successively with saturated $\mathrm{NaHCO}_{3}$, brine and water. The solution was then dried over anhydrous $\mathrm{Na}_{2} \mathrm{SO}_{4}$ and solvent removed under reduced pressure. The residue was purified by a short pad of silica gel (diethyl ether/hexane 1:10) to afford the phosphine $\mathbf{1 b}(0.21 \mathrm{~g})$. Yield: $90 \%$.

All the analytical data of the phosphines were identical as outlined above.

${ }^{18} \mathrm{O}$-labelling experiments. ${ }^{18} \mathrm{O}$-1a was prepared according to method a (vide supra) and reduced with the general procedure. HRMS $(70 \mathrm{eV})$, calcd $[\mathrm{M}+\mathrm{H}]$ for $\mathrm{C}_{52} \mathrm{H}_{25}{ }^{18} \mathrm{O}_{2} \mathrm{~F}_{24} \mathrm{P}_{2}$ : 1203.1026; found: 1203.1054 .

${ }^{18} \mathbf{O P P h}_{3}$ HRMS (70eV) calcd [M+H] for $\mathrm{C}_{18} \mathrm{H}_{15}{ }^{18} \mathrm{OP}: 281.0983$; found: 281.1013 . 


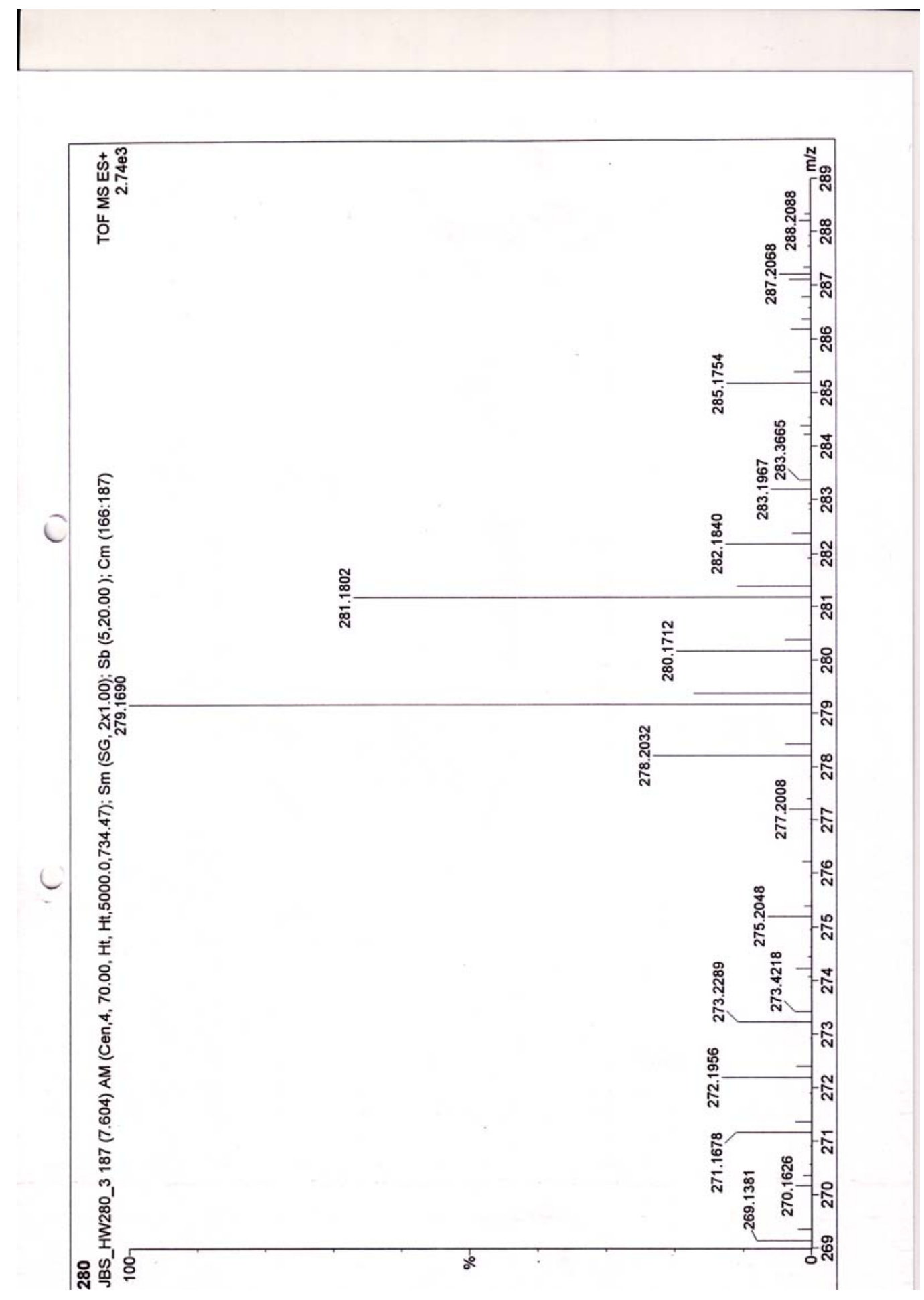

From the mass spectrum it clearly shows the ${ }^{18} \mathrm{O}$ transferred from the ${ }^{18} \mathrm{O}$-labeled substrate to triphenylphosphine. The presence of ${ }^{16} \mathrm{O}$ triphenylphosphine oxide was due to the aerobic workup conditions. 
X-ray crystal structures of phosphine oxides $\mathbf{1 a}, \mathbf{1 b}$ and phosphines $\mathbf{2 a}, \mathbf{2 b}$ (requisite CIF files have been submitted to the journal).

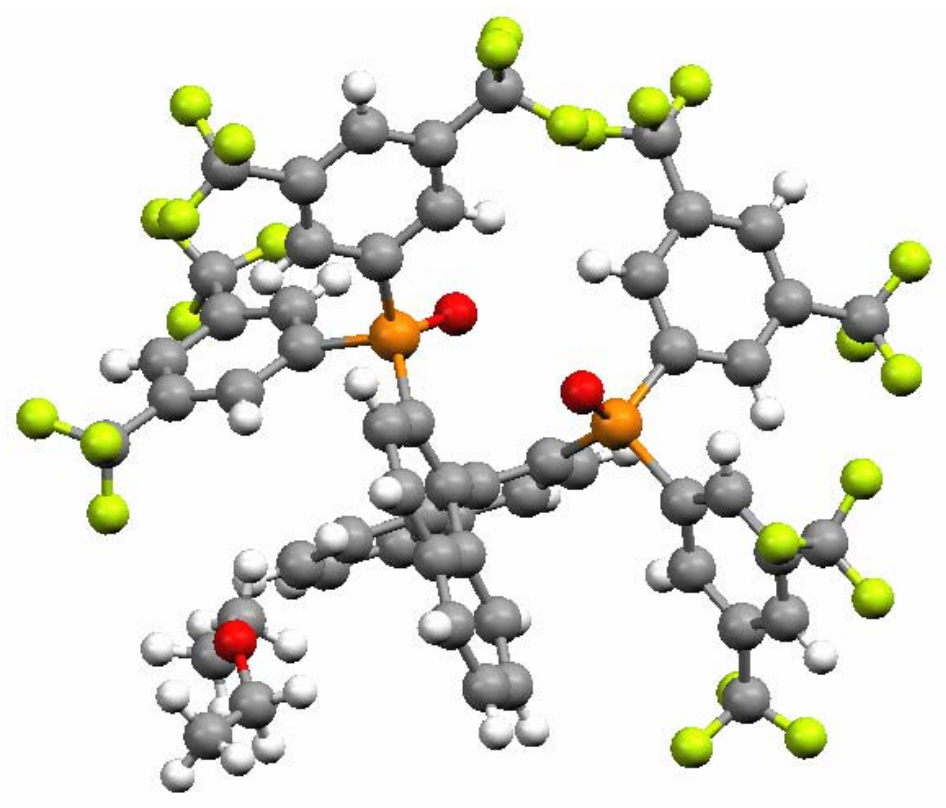

X-ray structure of 1a

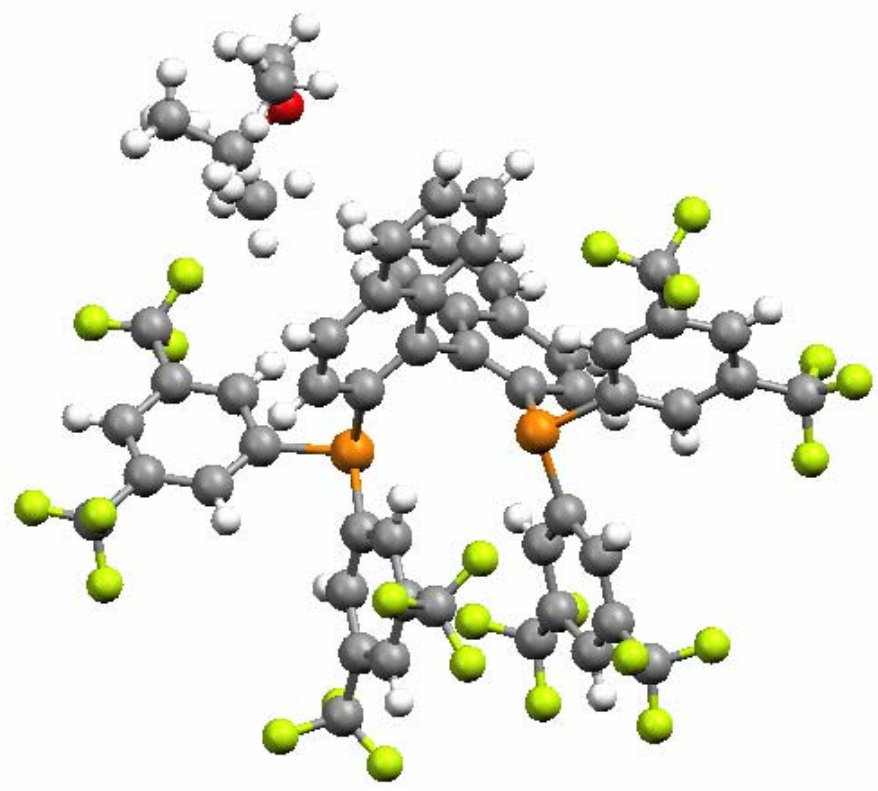

X-ray structure of $\mathbf{2 a}$ 


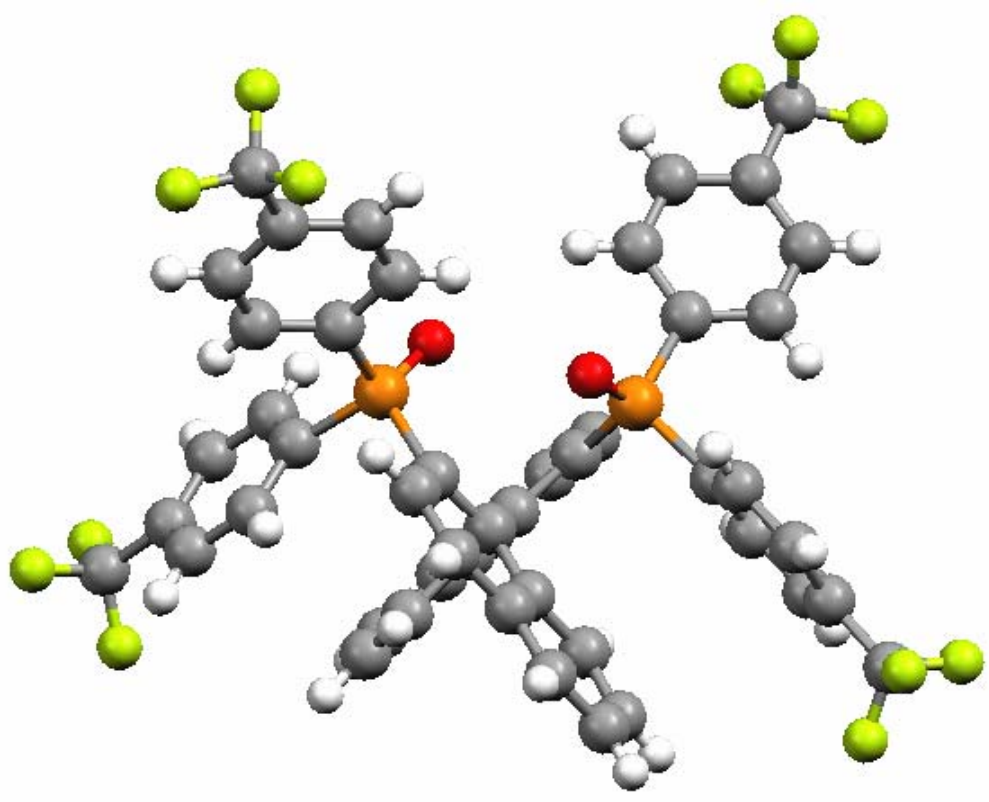

$\mathrm{X}$-ray structure of $\mathbf{1 b}$

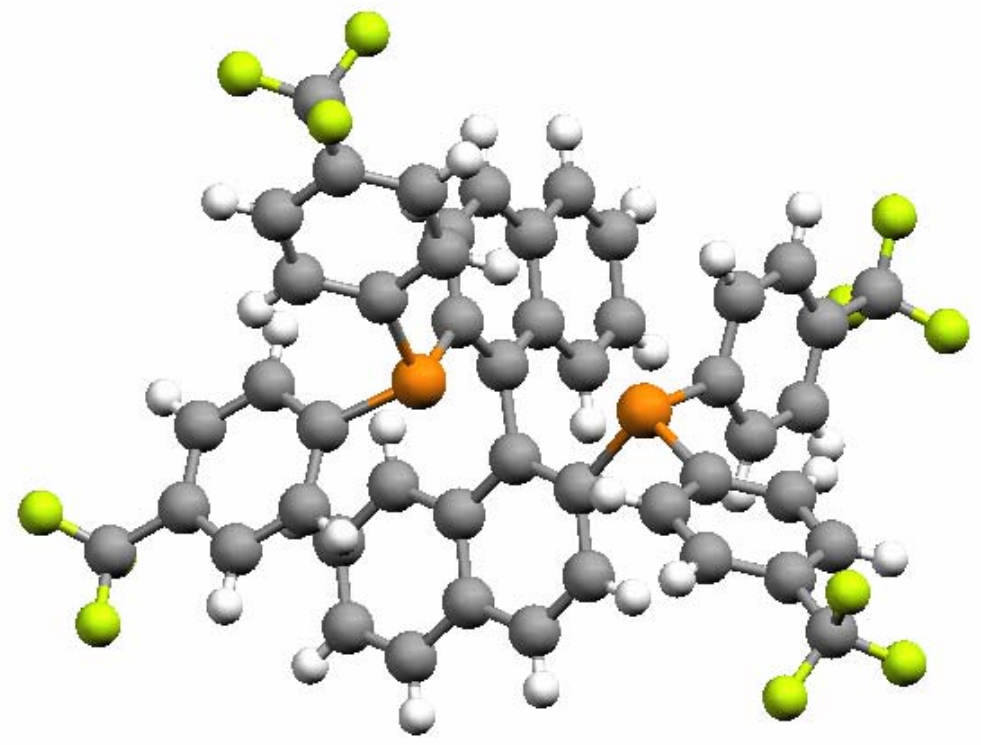

X-ray structure of $\mathbf{2 b}$ 


\section{Reference}

1 Takaya, H.; Mashima, K.; Koyano, K.; Yagi, M.; Kumobayshi, H.; Taketomi, T.; Akutagawa, S.; Noyori, R. J. Org. Chem. 1986, 51, 629.

2 Brown, J. K.; Berry, M. S.; Waterman, K. C.; Lingenfelter, D.; Murdoch, J. R . J. Am. Chem. Soc. 1984, 106, 4717.

3 Casalnuovo, A. L.; RajanBabu, T. V.; Ayers, T. A.; Warren, T. H. J. Am. Chem. Soc. 1994, 116, 9869.

4 Vineyard, B. D.; Knowles, W. S.; Sabacky, M. J.; Bachman, G. L.; Weinkauff, D. J. J. Am. Chem. Soc. 1977, 99, 5946.

5 Imamoto, T.; Kusumoto, T.; Suzuki, N.; Sato, K. J. Am. Chem. Soc. 1985, 107, 5301. 